BMJ Open

Diabetes

Research

\& Care

\title{
Association between regional deprivation and type 2 diabetes incidence in Germany
}

\author{
Esther Jacobs (D) , ${ }^{1,2}$ Thaddäus Tönnies, ${ }^{1}$ Wolfgang Rathmann, ${ }^{1,2}$ Ralph Brinks, ${ }^{1,3}$ \\ Annika Hoyer
}

To cite: Jacobs E, Tönnies T, Rathmann W, et al. Association between regional deprivation and type 2 diabetes incidence in Germany. BMJ Open Diab Res Care 2019;7:e000857. doi:10.1136/ bmjdrc-2019-000857

- Additional material is published online only. To view please visit the journal online (http://dx.doi.org/10.1136/ bmjdrc-2019-000857).

Received 27 August 2019 Revised 12 November 2019 Accepted 22 November 2019
Check for updates

C C Author(s) (or their employer(s)) 2019. Re-use permitted under CC BY-NC. No commercial re-use. See rights and permissions. Published by BMJ.

${ }^{1}$ Institute for Biometrics and Epidemiology, German Diabetes Center (DDZ), Leibniz Center for Diabetes Research at Heinrich Heine University Düsseldorf, Düsseldorf, Germany ${ }^{2}$ German Center for Diabetes Research (DZD), MünchenNeuherberg, Germany ${ }^{3}$ Hiller Research Unit for Rheumatology, Heinrich Heine University Düsseldorf, Düsseldorf, Germany

Correspondence to Ms Esther Jacobs; esther.jacobs@ddz.de

\section{ABSTRACT}

Objective The aim of this analysis was to estimate the association between regional deprivation and type 2 diabetes incidence and to investigate differences by age and sex for Germany.

Research design and methods Type 2 diabetes incidence rate ratios comparing the most deprived fifth of the population to the remainder of the population (divided into quintiles) were estimated using the illnessdeath model, which describes the relationship between prevalence, mortality, and incidence. For the analysis, we used the type 2 diabetes prevalence and the general mortality rate according to deprivation quintiles, which we calculated based on valid estimates for Germany. Because mortality rate ratios for people with type 2 diabetes compared with people without type 2 diabetes are lacking for Germany, we used estimates from Scotland. Estimates were standardized to the German population in 2012 and stratified by sex.

Results Incidence of type 2 diabetes was estimated to be over twice as high among people living in the most deprived regions of Germany compared with people living in the least deprived regions (men: $2.41,95 \% \mathrm{Cl} 1.27$ to 4.28; women: $2.40,95 \% \mathrm{Cl} 1.25$ to 4.29 ). The strength of the association increased with increasing age until the age of 75 years. No sex differences were present.

Conclusions The study adds new evidence regarding the association between type 2 diabetes incidence and regional deprivation for Germany. The results underpin the importance to intensify public health actions to reduce social inequalities in Germany and whole Europe in the future.

\section{INTRODUCTION}

The impact of the individual socioeconomic status (SES) and regional deprivation on behavior-related risk factors and prevalence of non-communicable diseases, such as type 2 diabetes, has become better understood in the last decades. ${ }^{1-6}$ Individual's education, income and occupation are the central defining factors of individual SES, whereas regional deprivation focuses more on the area-level indicators, such as unemployment rates. ${ }^{7}$ Existing evidence suggests that social inequality plays an important role both for prevalence and incidence of type

\section{Significance of this study}

What is already known about this subject?

- Evidence suggests that regional deprivation is associated with diabetes incidence, however, for Germany, instruments to measure regional deprivation have been developed but the association with diabetes incidence has not been analyzed so far.

What are the new findings?

- In Germany, living in areas with high regional deprivation is associated with a 2.4 times higher type 2 diabetes incidence rate than living in regions with low regional deprivation.

How might these results change the focus of research or clinical practice?

- The findings of our study show that regional deprivation is associated with diabetes incidence in Germany, and that the association is similar to other European countries. Thus, intensified public health actions are urgently needed to reduce social inequalities.

2 diabetes. ${ }^{38-11}$ In the past, individual SES and regional deprivation were often used as proxy for each other, however, some studies showed that both domains have an independent impact on type 2 diabetes prevalence and incidence. ${ }^{9} 12$

In Europe, several studies have shown that regional deprivation is associated with diabetes incidence. ${ }^{2} 11$ 13-18 For Germany, studies reported only diabetes incidence rates in different German regions based on regional cohort studies or claims data. ${ }^{19} 20$ Analyses regarding diabetes incidence using dimensions of regional socioeconomic inequalities, for example, multiple deprivation approaches, are lacking for Germany so far, although instruments to measure regional deprivation have been developed for Germany. ${ }^{4}$

Some studies investigating the association between diabetes incidence and social 
inequalities stated that higher age might diminish the strength of the association. ${ }^{11} 142122$ Studies presenting absolute rates found a higher association between social status and diabetes incidence in higher ages compared with younger ages. ${ }^{11} 1315$ A reduced or no statistically significant association in higher ages was found when ratios of deprivation, for example, high compared with low deprivation, were used. ${ }^{1421}$ Furthermore, sex differences have been reported. ${ }^{11} 13-151822$ However, not all analyses showed differences by sex or reached statistically significant differences between men and women. ${ }^{1821}$

Therefore, the aim of the present study was to estimate the sex-specific impact of regional deprivation on incidence of type 2 diabetes and to investigate differences in the impact of regional deprivation by age.

\section{PARTICIPANTS AND METHODS \\ Input data}

In order to estimate the association between regional deprivation and type 2 diabetes incidence we used available data as follows:

- The type 2 diabetes prevalence, stratified by regional deprivation $p^{(q)} \quad(\mathrm{p}=$ prevalence, $\mathrm{q}=$ deprivation according to quintiles), was calculated using quintilespecific ORs of the association between diabetes prevalence and area deprivation in Germany ${ }^{12}$ and the age and sex-specific prevalence of type 2 diabetes in Germany in 2009 and $2015 .^{20}$ The former underlying study from which we used the ORs is based on five population-based studies (study period $=1997-2006$, $\mathrm{n}=11.688$, age $=45-74)$ of the Diabetes Collaborative Research of Epidemiologic Studies, in which the German Index of Multiple Deprivation (GIMD) ${ }^{7}$ was used. The GIMD, originally developed in England and adapted for Germany, contains seven domains: income, employment, education, municipal revenue, social capital, environment, and security. Regional deprivation was determined at municipality level individually for each study participant. The municipalities were assigned to deprivation quintiles, in which a higher score indicates higher regional deprivation (quintile 1: least deprived; quintile 5: most deprived areas). ${ }^{7}$ Presented ORs were adjusted for individual SES, sex, age, body mass index (BMI) and lifestyle covariates. ${ }^{12}$ The latter study from which the prevalence of type 2 diabetes was taken is based on nationwide claims data from statutory health insurance including 69 million persons, representing outpatient care of about $85 \%$ of the German population. ${ }^{20}$

- The mortality rate ratio of people with and without diabetes stratified by regional deprivation $R^{(q)}$ is currently not available for Germany. Thus, we used information from a study conducted in Scotland, in which electronic records (study period $=2001-2007$, $\mathrm{n}=210.000$, age $=35-84$ ) from people with type 2 diabetes including information on the Scottish Index of Multiple Deprivation (distribution of quintiles as in ref 12) as well as mortality were available. ${ }^{23}$ The CIs of the stratified analysis by duration of diabetes ( $<2$ years vs $\geq 2$ years of diabetes duration) resulted in overlapping CIs, thus the point estimates and SEs were obtained using the geometric mean. Scotland/UK and Germany have a comparable standard of living and healthcare system. In 2012, the prevalence of diabetes (5.6\% in Germany, $4.8 \%$ in UK), ${ }^{24}$ mortality rates of people with diabetes (1.4 in Germany and 2.7 in UK per 100.000 deaths aged $0-64$ years $^{24}$ ) and of the general population are comparable (10.6 in Germany and 8.9 in UK per 1000 deaths $^{24}$ ). Furthermore, a number of examples in epidemiology showed that relative risks are stable measures across many different populations. ${ }^{25}$ Thus, we decided to use the estimated quintile-specific mortality rate ratios from Scotland for our analysis.

- The mortality ratio of the general population according to deprivation quintiles $m^{(q)}$ was calculated by using the general mortality $m$ for Germany ${ }^{26}$ and the estimates obtained in the socioeconomic panel (SOEP) study (1995-2005, $\mathrm{n}=32.000)$, containing annual survey data of adult household members. ${ }^{27}$ The relative mortality risk was presented in four income groups, taking into account the equivalized disposable income $(<60 \%, 60 \%-80 \%, 80 \%-100 \%$, $100 \%-150 \%$ of the mean income) which was compared with the highest income group $(>150 \%$ of the mean income) $\boldsymbol{R}_{m}^{(q)} \cdot{ }^{27}$ We assumed that the equivalized disposable income together with the information of the population proportion for each income group is a good proxy for deprivation. The general mortality ratio by deprivation quintiles $m^{q}$ was calculated as follows:

$$
m^{(q)}=R_{m}^{(q)} \frac{5 m}{\sum_{s=1}^{S} R_{m}^{(s)}}
$$

- The age pyramid for Germany in the year $2012^{28}$ was used to estimate age-standardized incidence of type 2 diabetes by sex and deprivation quintiles.

\section{Analysis}

To obtain the sex-specific incidence of type 2 diabetes by the quintiles of regional deprivation, we used an illnessdeath model, which describes the relationship between prevalence, mortality, and incidence. ${ }^{29}$ Therefore, the following differential equation ${ }^{29}$ describing this relationship was solved to calculate the incidence rate for each age $a$ at time by the deprivation quintiles $q$ :

$$
\left(\partial_{t}+\partial_{a}\right) p^{(q)}=\left(1-p^{(q)}\right)\left(i^{(q)}-m^{(q)} \frac{p^{(q)}\left(R^{(q)}-1\right)}{p^{(q)}\left(R^{(q)}-1\right)+1}\right)
$$

where:

$p^{(q)}$ is the type 2 diabetes prevalence stratified by regional deprivation, 
Men

Women

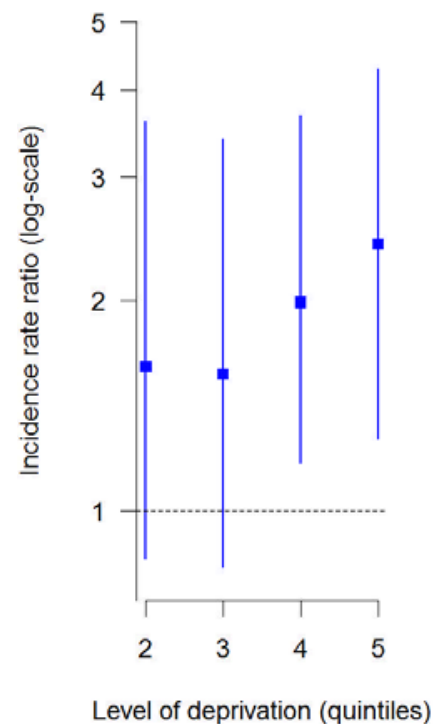

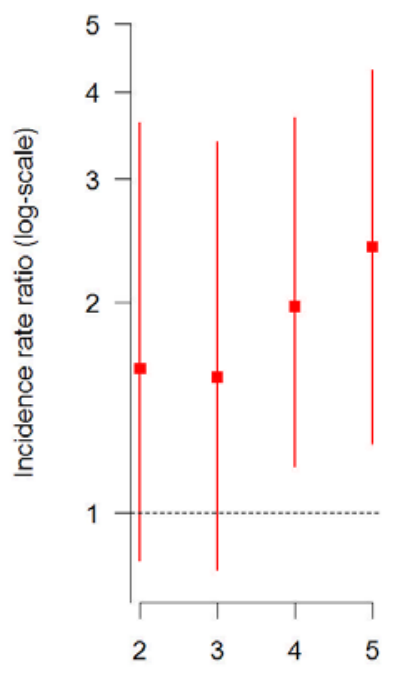

Level of deprivation (quintiles:

Figure 1 Age-standardized incidence rate ratios of type 2 diabetes $(95 \% \mathrm{Cls})$ in men and women by regional deprivation (quintiles 2-5 compared with the least deprived quintile 1) in Germany in 2012.

$i^{(q)}$ is the type 2 diabetes incidence stratified by regional deprivation,

$m^{(q)}$ is the mortality ratio of the general population according to deprivation quintiles,

$\boldsymbol{R}^{(q)}$ is the mortality ratio of people with and without diabetes, stratified by regional deprivation.

Additionally, we estimated the incidence of type 2 diabetes by regional deprivation for defined age groups $(20-44,45-64,65-74$ and $>74)$ as it is frequently used in other epidemiological studies. ${ }^{30}$

We estimated incidence rate ratios (IRR) by comparing the age-standardized incidence rates of the quintiles 2-5 with the least deprived quintile 1. Corresponding 95\% CIs were estimated using a Monte Carlo simulation. This means, from all confidence bounds of the input data (ie, type 2 diabetes prevalence, stratified by regional deprivation $p^{(q)}$, mortality rate ratio of people with and without diabetes stratified by regional deprivation $R^{(q)}$, and the mortality ratio of the general population according to deprivation quintiles $m^{(q)}$ ), we drew a sample according to a normal distribution and applied the estimation method for the IRR. This was repeated 5.000 times to estimate the distribution of the IRR. Based on this, we calculated the $95 \%$ confidence bounds of the IRR. Statistical analyses were performed by using R V.3.2.1 software (The R Foundation for Statistical Computing, Vienna, Austria). Because all data used have already been published, ethics committee approval was not necessary.

\section{RESULTS}

The association between regional deprivation and incidence of type 2 diabetes is shown in figure 1 and table 1 ,

in which the levels of deprivation (in quintiles) were compared with the least deprived level (quintiles 2-5 vs 1 ). For men and women living in areas with high regional deprivation (quintiles 4 and 5), the IRRs were significantly higher compared with those living in areas with low regional deprivation (eg, quintile 5 vs 1 in men: IRR 2.41; 95\% CI 1.27 to 4.28 ; women: 2.40 ; $95 \%$ CI 1.25 to 4.29) (figure 1, table 1). With increasing deprivation level, the IRR of type 2 diabetes increased both in men and women, however, the confidence intervals overlap to a great extent, which suggest that there is no evidence for sex differences between the quintiles of deprivation. Furthermore, no differences in age-standardized IRR between men and women were present.

In addition, we estimated the association between deprivation and type 2 diabetes incidence for defined age groups (20-44, 45-64, 65-74 and $>74$ years) (table 1 , online supplementary figure 1 ). With increasing age, IRR increased in all deprivation quintiles. Only in the age group $>75$ years, there was a tendency towards a reduced impact of regional deprivation in all deprivation quintiles, however, people living in high deprived areas (quintiles 4 and 5) still had a significantly higher IRR compared with those living in low deprived areas (quintile 1).

\section{DISCUSSION}

The present analysis, for which we used available epidemiological estimates and statistical data, showed the following results: First, incidence of type 2 diabetes was estimated to be over twice as high among people living in the most deprived regions of Germany compared with people living in the least deprived regions. Second, no differences between men and women exist in the relationship between deprivation and type 2 diabetes incidence. Third, in the age group of $>75$ years, the IRR for type 2 diabetes was slightly lower in people with high regional deprivation compared with the younger age groups.

For Germany, there are currently no studies regarding the association between diabetes incidence and regional socioeconomic inequalities published. Some studies investigated the association between regional deprivation and the prevalence of type 2 diabetes or diabetes risk factors for Germany. ${ }^{41012}$ For example, data from the TNS Health Care Access Panel in 2006 with 40.000 people from Germany and the representative German Health Update 'GEDA' telephone survey in 2009/2010 were used to assess the association of regional deprivation and type 2 diabetes prevalence or obesity in multivariate models. The age and sex-adjusted ORs of people living in the most deprived regions compared with people living in the least deprived regions were 1.66 $(95 \% \mathrm{Cl} 1.37$ to 2.00$)$ and $1.37(95 \% \mathrm{Cl} 1.19$ to 1.58$)$ for type 2 diabetes prevalence and $1.32(95 \% \mathrm{Cl} 1.19$ to $1.47)$ and $1.33(95 \% \mathrm{Cl} 1.18$ to 1.50$)$ for obesity, respectively. ${ }^{910}$ The adjustment for further risk factors, such as smoking, physical activity and BMI, diminished the association in all of the models somewhat, for example, from 
Table 1 Age-standardized and age-specific incidence rate ratios of type 2 diabetes ( $95 \%$ Cls) by regional deprivation in quintiles in Germany in 2012

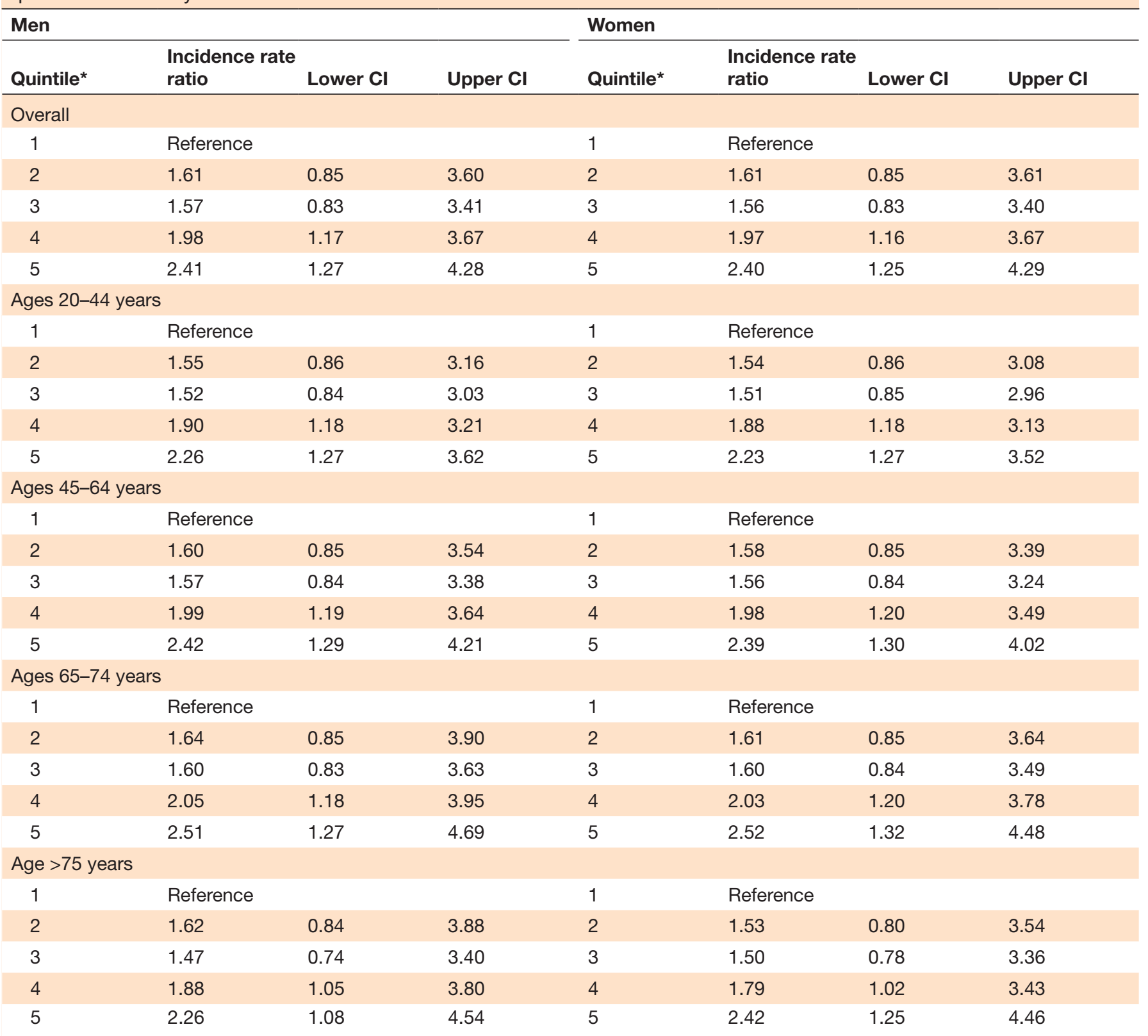

${ }^{*}$ Quintiles are compared with the least deprived areas (first quintile). The fifth quintile represents municipalities with the highest regional deprivation.

an OR of 1.37 to 1.18 for diabetes prevalence in the fully adjusted model including these risk factors in the GEDA sample. ${ }^{10}$ These results indicate a slightly lower association between regional deprivation and type 2 diabetes prevalence than incidence we found in our analysis. Furthermore, diabetes risk factors could fully explain this association. ${ }^{10}$ However, both studies are based on representative samples of the general population of Germany, thus, the studies might not represent all people with type 2 diabetes in Germany.

Similar to our study, previous studies from northern Europe indicate a strong association between regional deprivation and diabetes incidence (OR between 1.22 and 3.71). ${ }^{2151618}$ A study from Scotland based on the National
Diabetes Register revealed that between 2008 and 2013, incidence rates declined in the population except of the most deprived person groups. ${ }^{15}$ A cohort study from Finland, in which 3500 participants aged 6-18 years were followed up for 30 years, suggests that differences in lifestyle are already present in the years of childhood and adolescence. $^{2}$ This led to an OR of $3.71(95 \% \mathrm{Cl} 1.77$ to 7.75) for incident diabetes in adulthood in those with high neighborhood socioeconomic disadvantage, adjusted for covariates including individual SES. ${ }^{2}$ Another analysis from Sweden with 61000 refugees aged 25-50 years showed an OR of 1.22 (95\% Cl 1.07 to 1.38 ), adjusted for possible confounders including individual education level. ${ }^{16}$ It was also found that the strength of association between diabetes 
risk and high versus low regional deprivation increased over time, in 5 years by $9 \%{ }^{16}$

Type 2 diabetes incidence rates are higher in men than in women, especially in the middle age and higher age groups ( $\geq 40$ years of age).$^{1520}$ When considering rate ratios comparing quintiles of regional deprivation, we found slightly increasing IRR by age except of the highest age group of $>75$ years, in which the IRRs of all quintiles were somewhat lower, but with overlapping CIs. Furthermore, no evidence for differences between men and women was present in the analysis of an association between type 2 diabetes incidence and regional deprivation. Possibly, the impact of regional deprivation is lower in the oldest age group, because a high proportion of people receives support in daily life from nursing personnel or lives in care retirement homes where the regional deprivation is not such an important factor. Because death rates are higher in the older population and in regions with high deprivation, ${ }^{23}$ the presence of survival bias is also possible which means that socioeconomic differences may be reduced in the input data. However, the estimation method we used is based on simple algebraic transformations of an analytical relation from the illness-death model, which is why possible bias typically occurring in survival analysis is unlikely.

Other European studies, all based on routine medical health records, revealed heterogeneous results. A study from the UK found that especially older male patients living in regions with high deprivation had the highest risk to develop type 2 diabetes, however, incidence rates in the age group of 65-74 years were higher than in the age group of $>75$ years, which is in line with our results. ${ }^{11}$ A study from Sweden presented crude incidence rates by age group and gender by the level of regional deprivation. ${ }^{14}$ By calculating IRRs for high compared with low deprivation, a trend towards decreasing IRR by increasing age and a higher IRR in women compared with men becomes apparent. A study from Madrid, Spain also found a greater association between neighborhood deprivation and diabetes incidence in women than in men, adjusted for age. ${ }^{18}$ Women living in neighborhoods with low deprivation had a $31 \%$ lower hazard rate for diabetes incidence (HR 0.69; 95\% CI 0.59 to 0.80 ) compared with neighborhoods with high deprivation. In men, the difference was only $20 \%$ (HR $0.80 ; 95 \%$ CI 0.71 to 0.91$).{ }^{18}$ However, the CIs of men and women were overlapping, as we found in our analysis. It should be noticed that the results of the studies mentioned ${ }^{111418}$ were not adjusted for individual SES. In our analysis, the estimates on mortality and prevalence ratios used for the analysis were adjusted for possible confounders including individual SES, thus, differences between the studies are possible. Furthermore, the CIs in our analysis remained relatively wide due to the assumptions we needed to make, thus the real effect sizes can slightly differ.

In addition to existing evidence on the association between SES and regional deprivation on diabetes incidence and prevalence, ${ }^{3}{ }^{8-11}$ studies indicate that the effect of deprivation on diabetes risk increases over time. ${ }^{2} 16$ Moreover, lifestyle factors, that is, smoking, alcohol intake, physical activity, and BMI, explain up to $52 \%$ of the differences in diabetes risk by SES, as shown in the English Longitudinal Study of Ageing. ${ }^{5}$ Taken this evidence together, it is important to intensify public health actions to reduce social inequalities in the future. A good starting point is the concept of Europe 2020, the European policy for health and well-being, which was approved by the WHO in 2012. ${ }^{1}$ The aim is to tackle inequities and the social determinants of health. ${ }^{1}$ This includes structural changes to reduce poverty, for example, a higher minimum wage and higher taxes on high incomes and high profit companies and the development of living environments that supports a healthy lifestyle. Furthermore, primary prevention programs with a focus on reducing diabetes risk factors are needed predominantly in regions with high deprivation to improve health education.

\section{Strength and weaknesses}

The major strength of our study is that the approach we used enabled us to assess the association between nationwide diabetes incidence and regional deprivation in Germany for the first time. Because no nationwide diabetes register is implemented in Germany so far, opportunities for population-based epidemiological analyses in the field of diabetes such as the present one are restricted to routine data, regional cohort studies, or representative surveys for the general population (with only few people with diabetes included). For our analysis, which is based on the illness-death model, we used solely valid estimates, such as the information on differences in type 2 diabetes prevalence by area deprivation measured using the validated German Index of Socioeconomic Deprivation. ${ }^{12}$ Simultaneously, it was necessary to make a number of assumptions, which resulted in wide CIs, which is the major weakness. For example, estimates on mortality of the general population according to quintiles of deprivation were needed. The only study that was available for Germany was a study based on the SOEP in which the equivalized disposable income was used to estimate differences in mortality by socioeconomic differences. ${ }^{27}$ Moreover, in the Scottish study ${ }^{23}$ we used for mortality by regional deprivation, the quintiles were defined at a national level. This means the deprivation quintiles could vary between Scotland and Germany. However, the Scottish and German population is comparable, as described in the Participants and Methods section, which means that big differences in regional deprivation quintiles are unlikely. Furthermore, examples in epidemiology exist showing that relative risks are stable measures across many different populations. ${ }^{25}$

In our analysis, we assessed type 2 diabetes IRRs comparing higher regional deprivation to low regional deprivation. The results show that in Germany, type 2 diabetes incidence differs by regional deprivation and that the strength of the association increases with 
increasing age until the age of about 75 years. Furthermore, we did not find sex differences. The study adds new evidence regarding the association of type 2 diabetes incidence and regional deprivation for Germany and underpins the importance of public health measures to reduce social inequality. Ideally, future studies should also focus on the impact of regional deprivation on type 2 diabetes incidence in the older population.

Contributors All authors gave important intellectual contributions, reviewed the manuscript and gave final consent to the version to be published. EJ contributed to the discussion, interpreted the results and wrote the manuscript. TT contributed to the discussion and reviewed the manuscript. WR reviewed the manuscript. RB and AH developed the study plan, contributed to the discussion, analyzed the data and reviewed the manuscript. AH is the guarantor of this work and, as such, had full access to all the data in the study and takes responsibility for the integrity of the data and the accuracy of the data analysis.

Funding This analysis was conducted at the German Diabetes Center (DDZ), which is funded by the German Federal Ministry of Health and the Ministry of Innovation, Science, Research and Technology of the State North Rhine-Westphalia. This analysis was further supported by a grant from the German Federal Ministry of Education and Research (BMBF) to the German Center for Diabetes Research (DZD).

Competing interests WR reported receiving consulting fees for attending educational sessions or advisory boards from AstraZeneca, Boehringer Ingelheim and Novo Nordisk and institutional research grants from Novo Nordisk.

Patient consent for publication Not required.

Provenance and peer review Not commissioned; externally peer reviewed.

Data availability statement All data relevant to the study have already been published and are cited in the article.

Open access This is an open access article distributed in accordance with the Creative Commons Attribution Non Commercial (CC BY-NC 4.0) license, which permits others to distribute, remix, adapt, build upon this work non-commercially, and license their derivative works on different terms, provided the original work is properly cited, appropriate credit is given, any changes made indicated, and the use is non-commercial. See: http://creativecommons.org/licenses/by-nc/4.0/.

ORCID IDs

Esther Jacobs http://orcid.org/0000-0002-2200-4024

Annika Hoyer http://orcid.org/0000-0002-0241-9951

\section{REFERENCES}

1 World Health Organization (WHO). Health 2020: the European policy framework and strategy of the 21st century, 2013. Available: http:// www.euro.who.int/_data/assets/pdf_file/0011/199532/Health2020Long.pdf?ua $=1$ [Accessed 17 July 2019].

2 Kivimäki M, Vahtera J, Tabák AG, et al. Neighbourhood socioeconomic disadvantage, risk factors, and diabetes from childhood to middle age in the young Finns study: a cohort study. Lancet Public Health 2018;3:e365-73.

3 Heidemann C, Du Y, Baumert J, et al. Social inequality and diabetes mellitus - developments over time among the adult population in Germany. J Health Monitor 2019;4:11-28.

4 Kroll LE, Schumann M, Hoebel J, et al. Regional health differences developing a socioeconomic deprivation index for Germany. J Health Monitor 2017;2.

5 Stringhini S, Zaninotto P, Kumari M, et al. Lifecourse socioeconomic status and type 2 diabetes: the role of chronic inflammation in the English longitudinal study of ageing. Sci Rep 2016;6:24780.

6 Williams ED, Magliano DJ, Zimmet PZ, et al. Area-level socioeconomic status and incidence of abnormal glucose metabolism: the Australian diabetes, obesity and lifestyle (AusDiab) study. Diabetes Care 2012;35:1455-61.

7 Maier W, Fairburn J, Mielck A. Regional Deprivation and Mortality in Bavaria. Development of a Community-Based Index of Multiple Deprivation]. Gesundheitswesen 2012;74:416-25.

8 Agardh E, Allebeck P, Hallqvist J, et al. Type 2 diabetes incidence and socio-economic position: a systematic review and metaanalysis. Int J Epidemiol 2011;40:804-18.
9 Grundmann N, Mielck A, Siegel M, et al. Area deprivation and the prevalence of type 2 diabetes and obesity: analysis at the municipality level in Germany. BMC Public Health 2014:14:1264-64.

10 Maier W, Scheidt-Nave C, Holle R, et al. Area Level Deprivation Is an Independent Determinant of Prevalent Type 2 Diabetes and Obesity at the National Level in Germany. Results from the National Telephone Health Interview Surveys 'German Health Update' GEDA 2009 and 2010. PLoS One 2014;9:e89661.

11 Zghebi SS, Steinke DT, Carr MJ, et al. Examining trends in type 2 diabetes incidence, prevalence and mortality in the UK between 2004 and 2014. Diabetes Obes Metab 2017;19:1537-45.

12 Maier W, Holle R, Hunger M, et al. The impact of regional deprivation and individual socio-economic status on the prevalence of Type 2 diabetes in Germany. A pooled analysis of five population-based studies. Diabetic Medicine 2013;30:e78-86.

13 Cox M, Boyle PJ, Davey PG, et al. Locality deprivation and type 2 diabetes incidence: a local test of relative inequalities. Soc Sci Med 2007;65:1953-64.

14 Mezuk B, Chaikiat Åsa, Li X, et al. Depression, neighborhood deprivation and risk of type 2 diabetes. Health Place 2013;23:63-9.

15 Read SH, Kerssens JJ, McAllister DA, et al. Trends in type 2 diabetes incidence and mortality in Scotland between 2004 and 2013. Diabetologia 2016;59:2106-13.

16 White JS, Hamad R, Li X, et al. Long-Term effects of neighbourhood deprivation on diabetes risk: quasi-experimental evidence from a refugee dispersal policy in Sweden. Lancet Diabetes Endocrinol 2016;4:517-24.

17 Bilal U, Glass TA, Del Cura-Gonzalez I, et al. Neighborhood social and economic change and diabetes incidence: the HeartHealthyHoods study. Health Place 2019;58:102149.

18 Bilal U, Hill-Briggs F, Sánchez-Perruca L, et al. Association of neighbourhood socioeconomic status and diabetes burden using electronic health records in Madrid (Spain): the HeartHealthyHoods study. BMJ Open 2018;8:e021143.

19 Schipf S, Ittermann T, Tamayo T, et al. Regional differences in the incidence of self-reported type 2 diabetes in Germany: results from five population-based studies in Germany (DIAB-CORE Consortium). J Epidemiol Community Health 2014;68:1088-95.

20 Goffrier B, Schulz M, Bätzing-Feigenbaum J. Administrative Prävalenzen und Inzidenzen des Diabetes mellitus von 2009 bis 2015 [in German]. Versorgungsatlas-Bericht 2017;03.

21 Kowall B, Rathmann W, Strassburger K, et al. Socioeconomic status is not associated with type 2 diabetes incidence in an elderly population in Germany: KorA S4/F4 cohort study. J Epidemiol Community Health 2011;65:606-12.

22 Wray LA, Alwin DF, McCammon RJ, et al. Social status, risky health behaviors, and diabetes in middle-aged and older adults. $J$ Gerontol B Psychol Sci Soc Sci 2006;61:S290-8.

23 Walker JJ, Livingstone SJ, Colhoun HM, et al. Effect of socioeconomic status on mortality among people with type 2 diabetes. A study from the Scottish Diabetes Research Network Epidemiology Group 2011;34:1127-32.

24 World Health Organization (WHO). European health information gateway, 2019. Available: https://gateway.euro.who.int/en/hfaexplorer/ [Accessed 16 Jul 2019].

25 Breslow NE, Day NE. Fundamental Measures of Disease Occurrence and Association. Statistical Methods in Cancer Research, Volume 1-The Analysis of Case-Control Studies. In: IARC scientific publications No. 32: international agency for research on cancer, 1980: 42-83.

26 Federal Statistical Office. Life table (period life table), table 126210001, Sterbetafel (Periodensterbetafel): Deutschland, Jahre, Geschlecht, Vollendetes Alter [in German], 2012. Available: https:// www-genesis.destatis.de [Accessed 28 May 2019].

27 Kroll LE, Lampert T. Socio-Economic Differences in Life Expectancy: Data Sources in Germany and the Potential of the German Socio-Economic Panel Study]. Methoden, Daten, Analysen 2009;3:3-30

28 Federal Statistical Office. Population in Germany 2012 based on census, 2011. Available: https://service.destatis.de/bevoelkerung spyramide/index.html\#!y=2012\&v=2 [Accessed 15 July 2019].

29 Brinks R, Landwehr S. A new relation between prevalence and incidence of a chronic disease. Mathematical Med Biol 2015;32:dqu024-35.

30 Gregg EW, Cheng YJ, Srinivasan M, et al. Trends in cause-specific mortality among adults with and without diagnosed diabetes in the USA: an epidemiological analysis of linked national survey and vital statistics data. The Lancet 2018;391:2430-40. 